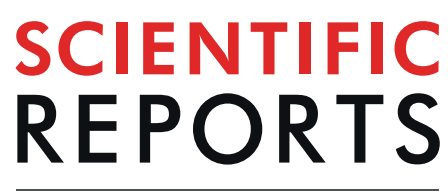

natureresearch

\title{
OPEN Compact all-fiber source of coherent linearly polarized octave- spanning supercontinuum based on normal dispersion silica fiber
}

Received: 13 June 2019

Accepted: 12 August 2019

Published online: 23 August 2019

\begin{abstract}
Karol Tarnowski ${ }^{1}$, Tadeusz Martynkien ${ }^{1}$, Paweł Mergo ${ }^{2}$, Jarosław Sotor ${ }^{3}{ }^{3}$ \& Grzegorz Soboń (iD
We report the generation of coherent octave-spanning supercontinuum in an all-fiber system, without any free-space optical components. The setup uses the femtosecond fiber laser as a pump and an allnormal dispersion microstructured fiber as a medium for supercontinuum generation. The generated spectrum is characterized both experimentally and numerically and shows a broad bandwidth $(1.1-2.2 \mu \mathrm{m})$, a high signal to noise ratio reaching 100 at maximum, a high coherence (closing to 1 ), linear polarization and average output power up to $57 \mathrm{~mW}$. The source is characterized by exceptional simplicity and does not require any alignment (the nonlinear fiber is spliced to the pump) which finally opens the path to outside-lab applications of supercontinuum radiation.
\end{abstract}



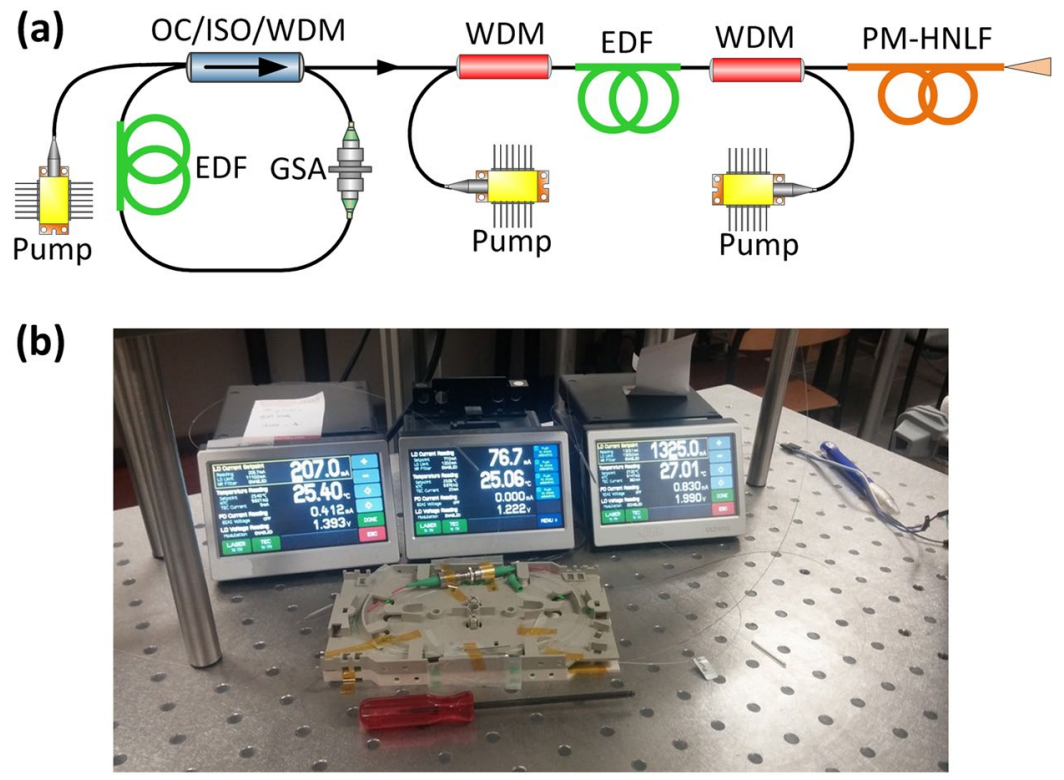

Figure 1. (a) Experimental setup of the all-fiber ANDi-SC source. EDF: Erbium-doped fiber. GSA: graphene saturable absorber. OC: output coupler, ISO: isolator, WDM: wavelength division multiplexer, PM-HNLF: polarization maintaining highly nonlinear fiber. (b) Photograph of the device running in the lab. The oscillator and amplifier are placed in two standard telecom trays (laser diode drivers are in the back).

stage to convert laser pulse into Raman soliton centered at $1.67 \mu \mathrm{m}$, which was filtered with a low pass filter and used as the pump for SC generation process. The octave spanning supercontinuum was generated by Nozaki ${ }^{18}$ in a dispersion shifted fiber (the spectrum from $0.95 \mu \mathrm{m}$ to $2.30 \mu \mathrm{m}$ and in a normal dispersion fiber (the spectrum from $1.05 \mu \mathrm{m}$ to $2.15 \mu \mathrm{m}$ ). However, the presented system is not all-fiber again.

Keeping the system entirely fiberized has multiple advantages. First of all, it is completely alignment-free. Secondly, it can be built entirely from polarization maintaining (PM) fibers, which ensures a linear polarization state at the output and invulnerability to external disturbances. Finally, it significantly reduces the costs of the system, by avoiding free-space optics (telescopes, objectives) and micrometer stages used for alignment. These are important features from the application point of view. To the best of our knowledge, coherent and all-normal dispersion generation of SC was not demonstrated in a fully fiberized compact system. Developing such compact and robust source could increase the scope of applications of ANDi-SC. The SC sources based on microstructured fibers were successfully applied for example in a confocal microscope $\mathrm{e}^{19}$, in an optical coherence tomography ${ }^{20}$ and in the measurement of optical frequencies ${ }^{21}$.

In this work, we present an all-fiber source of coherent and linearly polarized octave-spanning supercontinuum generated in an all-normal dispersion fiber. We used a femtosecond Er-doped fiber laser as a pump source and spliced it directly with the SC fiber. Importantly, the silica SC fiber is fully compatible with telecom-grade single-mode fibers, so it does not require any sophisticated splicing techniques. The generated SC has an average power of $57 \mathrm{~mW}$ (at $45 \mathrm{MHz}$ repetition rate) and spans over one octave $(1.1-2.2 \mu \mathrm{m}$ ). We have confirmed the high coherence and excellent shot-to-shot stability both experimentally and numerically. We believe that the presented approach paves the way to outside-lab applications of SC sources in harsh environment.

\section{Results and Discussion}

The experimental setup of the coherent supercontinuum source is presented in Fig. 1 . The system is built of only three fiber optic components and three pump lasers. An Er-doped fiber laser (EDFL) followed by an amplifier is used as the pump source for SC generation. The EDFL is based on a hybrid component, which comprises an output coupler (OC), isolator (ISO) and wavelength-division multiplexer (WDM) in one integrated package. The oscillator is mode-locked with a graphene saturable absorber (GSA). After amplification, the average output power reaches $116 \mathrm{~mW}$ at $45 \mathrm{MHz}$ repetition frequency, which corresponds to a pulse energy of approx. $2.6 \mathrm{~nJ}$. The pulse duration is at the level of $25 \mathrm{fs}$. The optical spectrum and pulse autocorrelation are depicted in Fig. 2. A small pedestal is visible in the pulse autocorrelation and we performed fitting of the autocorrelation assuming that there are two subpulses that accompany the main pulse as described in Methods. The details on the fiber laser can be found in ref. ${ }^{22}$. The polarization maintaining highly nonlinear fiber (PM-HNLF) used for SC generation is directly spliced to the output of the amplifier. The entire source is based on standard, PM single-mode fibers (PM-SMF) and components. Since the PM-HNLF is a silica fiber with a solid glass core, it is easily spliceable to the PM-SMF with reasonable loss (approx. $2 \mathrm{~dB}$ ) without using sophisticated techniques incorporating intermediate fibers or expensive filament splicers. It is worth noting that the system works also with only one pumping diode in the amplifier (as described $i^{22}$ ). The generated supercontinuum is however slightly narrower and does not cover one octave, nevertheless, it also maintains high coherence and all other stability features. The output port of the system can be easily connectorized by splicing a PM pigtail. 

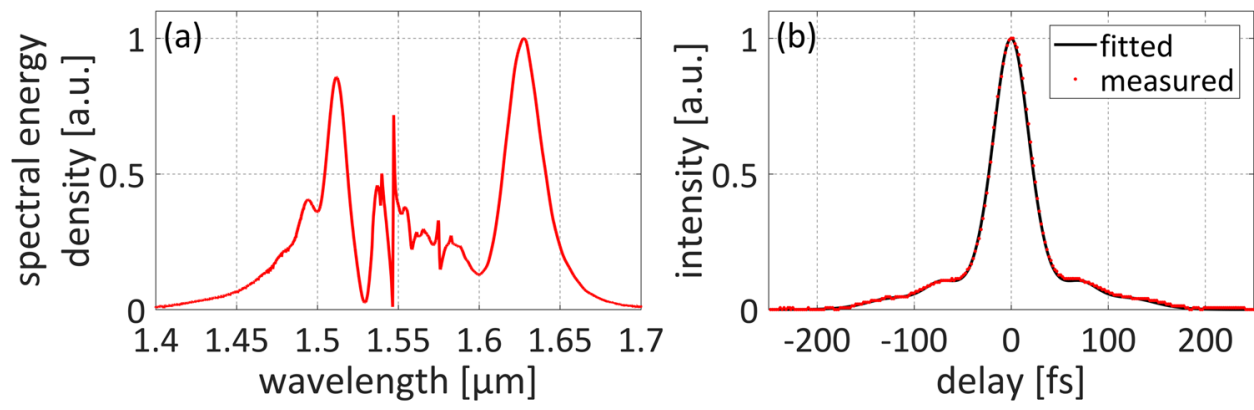

Figure 2. Measured pump pulse characteristics: (a) optical spectrum, (b) autocorrelation trace (the fitting parameters are gathered in Table 1 in section Methods).
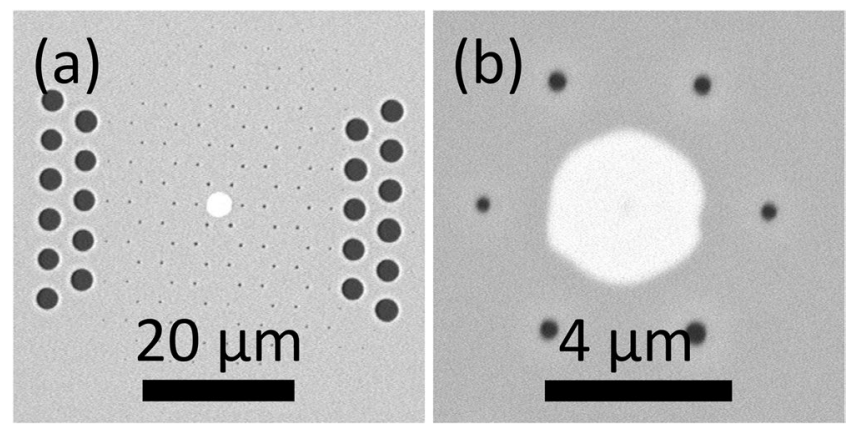

Figure 3. SEM images of microstructured fiber cross-sections: (a) core-cladding microstructure, (b) zoom on the core area.

The PM-HNLF used for SC generation is a silica microstructured fiber with a germanium doped core, which exhibits all-normal dispersion ${ }^{12}$. Scanning electron microscope (SEM) images of the fiber's cross section are presented in Fig. 3. The fiber's preform was stacked with a germanium-doped rod (core) and pure silica rods and capillaries (cladding). The capillaries were grouped symmetrically on two sides of the core (Fig. 3(a)). This alignment allows to induce lattice squeezing during fiber drawing and in consequence to force birefringence. The same approach was successfully applied in $^{13}$. The germanium-doped area (doping level of $18 \mathrm{~mol} \%$ ) has a hexagonal shape. The equivalent ellipse (in terms of normalized second central moments) has the major axis of $3.37 \mu \mathrm{m}$ and the minor axis of $3.22 \mu \mathrm{m}$, showing slight squeeze. The diameter of the first air holes ring is estimated to $6.2 \mu \mathrm{m}$ and the averaged diameter of the air holes is $0.38 \mu \mathrm{m}$. The measured and calculated properties of the fiber are presented in Fig. 4. The comparison of measured and calculated group velocity dispersions (GVD, $\left.\beta_{2}\right)$ is depicted in Fig. 4(a,b). The GVD reaches the minimum value of $0.8 \mathrm{ps}^{2} / \mathrm{km}$ around $1.7 \mu \mathrm{m}$ (what corresponds to the maximum chromatic dispersion $D=-0.5 \mathrm{ps} / \mathrm{km} / \mathrm{nm}$ ). The fiber exhibits group birefringence at the level of $10^{-5}$, as can be seen in Fig. 4(c). As it will be shown later, this birefringence level allows to maintain linear polarization of the generated SC. The calculated effective mode area keeps below $30 \mu \mathrm{m}^{2}$ in the entire range of interest, as plotted in Fig. 4(d), and takes the value of $14 \mu \mathrm{m}^{2}$ at $1.55 \mu \mathrm{m}$.

The supercontinuum generation takes place on $73 \mathrm{~cm}$ of fiber length. The spectrum covers one octave from $1.1 \mu \mathrm{m}$ to $2.2 \mu \mathrm{m}$ as shown in Fig. 5(a) (black line) and the average output power measured directly after the HNLF (without the output pigtail) is $57.2 \mathrm{~mW}$. After splicing an additional connector the power is at the level of $45 \mathrm{~mW}$. The calculated output spectrum is presented in Fig. 5(a) with green line and is in qualitative agreement with measured spectrum in terms of width and flatness. The calculations are based on a self-developed Nonlinear Schrödinger Equation (NLSE) solver. We attribute the low flatness of the generated SC to the non-ideal shape of the input pump pulse. As can be seen Fig. 2(b) and described in Methods, the pulse contains a pedestal which can be fitted as a superposition of three subpulses. It was confirmed in ${ }^{12}$ that the non-ideal shape induces degradation of the flatness. Additionally, the dips around $1.4 \mu \mathrm{m}$ and $2.2 \mu \mathrm{m}$ are related to $\mathrm{OH}$ absorption bands.

To verify the stability of the source, the SC was characterized with dispersive Fourier transform (DFT) technique, commonly used to investigate the shot-to-shot noise of SC sources ${ }^{23,24}$. Figure 5(b) presents imposed temporal traces of 500 DFT shots (grey dots) and their average (red line). The inset shows time-wavelength mapping applied to restore spectral dependence of individual pulses and their average presented in Fig. 5(a) with grey dots and red line. The wavelength range of DFT is limited by the properties of stretching fiber. The stretching fiber has a zero dispersion wavelength at $1.29 \mu \mathrm{m}$, as a result the time-wavelength mapping is not unique below $1.425 \mu \mathrm{m}$. On the other hand, the stretching fiber is attenuating beyond $1.9 \mu \mathrm{m}(10 \mathrm{~dB}$ at $1.94 \mu \mathrm{m},>20 \mathrm{~dB}$ at wavelengths $>2.0 \mu \mathrm{m}$ ) and as a result the long wavelength limit of DFT is around $1.9 \mu \mathrm{m}$. The comparison shown in Fig. 5(a) proves that spectrum registered with OSA and obtained with DFT are in excellent agreement in the $1.425-1.9 \mu \mathrm{m}$ range, which confirms the proper time-wavelength mapping. What can be also seen from Fig. 5 is 




$\begin{array}{llllllll}0.8 & 1 & 1.2 & 1.4 & 1.6 & 1.8 & 2 & 2.2\end{array}$ wavelength $[\mu \mathrm{m}]$

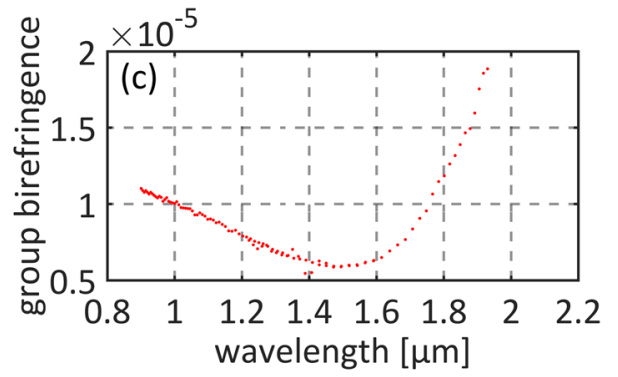

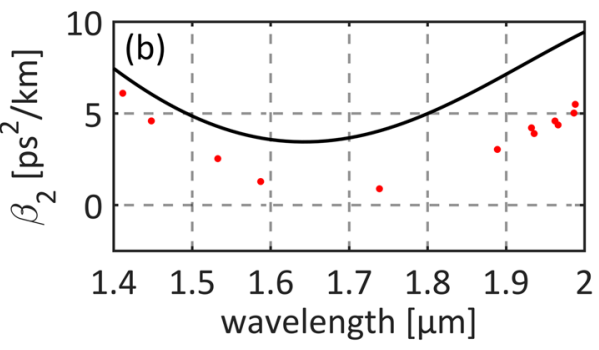



Figure 4. Characteristics of the PM-HNLF: (a) group delay dispersion; dotted line: measurement, solid line: calculation based on SEM image, (b) group delay dispersion zoom to 1.4-2.0 $\mu \mathrm{m}$ range (c) measured group birefringence, $(\mathbf{d})$ calculated effective mode area.
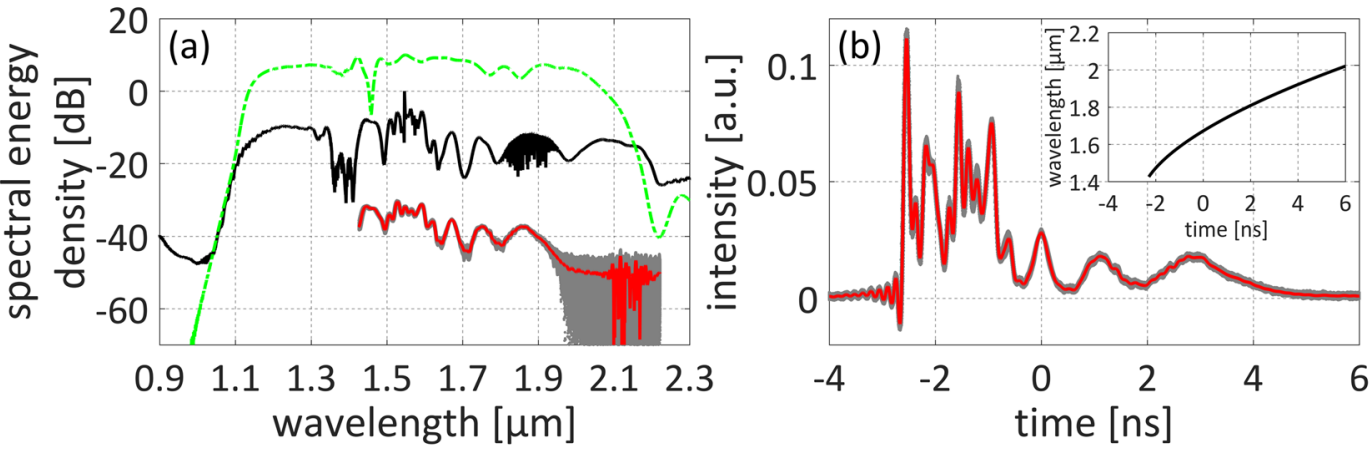

Figure 5. Characteristics of generated SC spectrum: (a) comparison of spectrum registered with OSA (black), spectrum calculated with NLSE (green) and spectrum retrieved with DFT: grey dots and red line (single shots and averaged spectrum, respectively); (b) temporal traces of 500 shots and their average grey dots and red line (single shots and their average, respectively); the inset shows time-wavelength mapping.

that the generated SC is characterized by very good stability and very low shot-to-shot fluctuations, comparable to ANDi-SC generated in soft glass fibers ${ }^{24}$. However, in all previous experiments, the fibers were free-space coupled with the pumping sources, which makes them useless in real-life applications.

The coherence properties of the generated SC were investigated both numerically and experimentally. We calculated the spectral coherence $\mathrm{g}_{12}{ }^{25}$ by performing 500 simulations with random noise (one-photon per mode) using the NLSE solver ${ }^{25-27}$. The result presented in Fig. 6(a) shows that generated spectrum maintains coherence in the entire covered range. To confirm high coherence experimentally, we performed measurements of pulse-to-pulse interference using a fiber-based unequal-path Michelson interferometer ${ }^{28,29}$. The interference pattern of two consecutive pulses of the train is shown in Fig. 6(b,c) with black dots. The shaded area in Fig. 6(b) corresponds to $1.65-1.7 \mu \mathrm{m}$ range, which is zoomed in Fig. 6(c), to reveal individual fringes. The upper (red) and bottom (blue) envelopes of the spectra were used for calculation of the fringe visibility function ( $V$, green), which can be directly referred to coherence ${ }^{24,28,29}$. The measurement confirms that the generated SC is highly coherent. The interference pattern was observed in slightly limited spectral range, due to limited operation range of the $50 / 50 \%$ fiber coupler used in the interferometer setup (originally designed for the $1.55 \mu \mathrm{m}$ window), nevertheless the interference pattern visibility is higher than 0.7 in $1.23-1.88 \mu \mathrm{m}$ range. The visibility is also limited by the presence of $\mathrm{OH}$ absorption band at $1.89 \mu \mathrm{m}$ and higher order modes below $1.3 \mu \mathrm{m}$. The visibility distribution over the entire observed bandwidth is not flat mainly due to the non-equal splitting ratio of the used fiber coupler for wavelengths $>1.7 \mu \mathrm{m}$ and $<1.4 \mu \mathrm{m}$.

We also took advantage of the DFT measurement and calculated the signal to noise ratio (SNR, defined as a ratio between mean and standard deviation at given wavelength) and the correlation maps of the generated SC. Both parameters are often used to analyze shot-to-shot stability of SC sources ${ }^{24,30}$. The SNR vs. wavelength plot is 

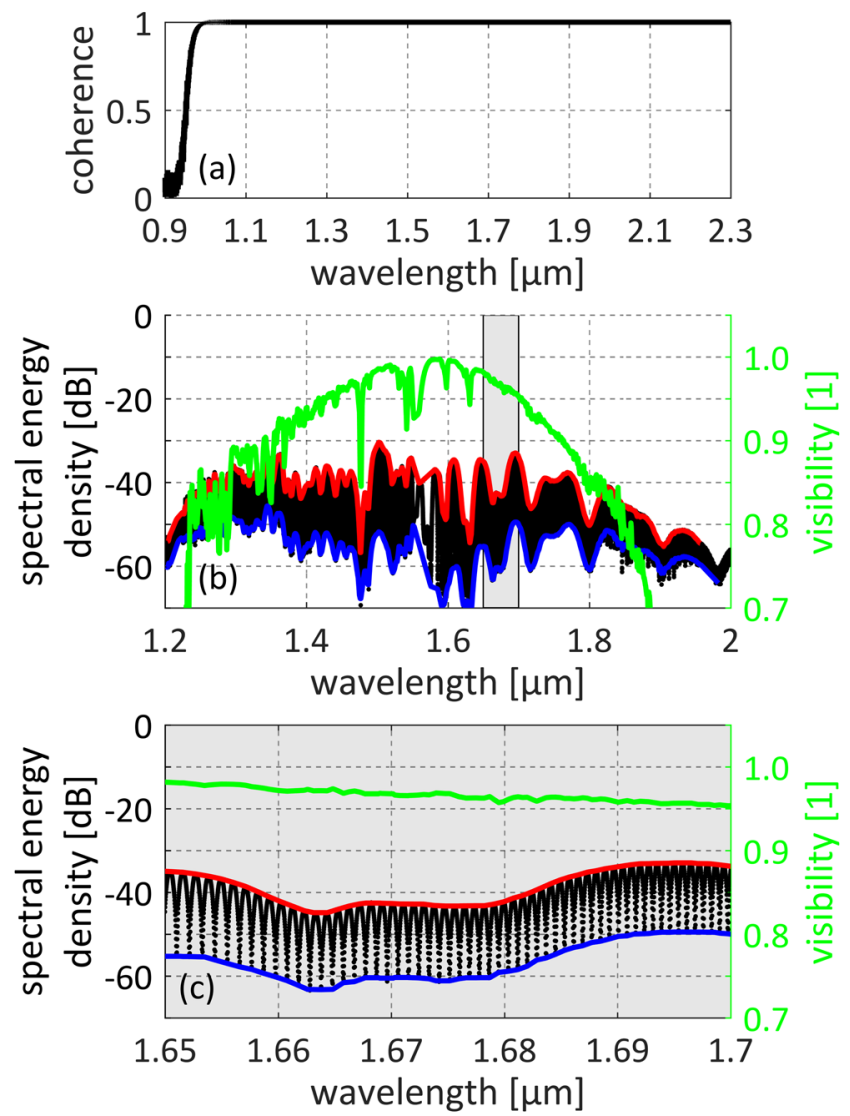

Figure 6. Results of coherence calculations and measurements: (a) coherence calculated with NLSE solver, (b) measured pulse-to-pulse interference and calculated fringes visibility, (c) measured pulse-to-pulse interference and calculated visibility zoomed in $1.65-1.7 \mu \mathrm{m}$ range.



Figure 7. Signal to noise ratio measured with DFT technique in $1.425-1.9 \mu \mathrm{m}$ range.

presented in Fig. 7. and the correlation map presented in Fig. 8. The obtained results confirm high signal-to-noise ratio, beyond 10 in almost entire spectral range, with maximum value of $\sim 100$. The SNR values are close to obtained for SC generated in soft glass $\mathrm{PCF}^{24}$.

The stability, noise and shot-to-shot dynamics of SC generation can be studied by using a wavelength correlation map, which describes how the pairs of wavelengths in the spectrum are correlated with each other. The wavelength correlation function, as defined $i^{30}$, varies between -1 and 1 . A positive correlation (red color in Fig. 8) between two chosen wavelengths from the $\mathrm{x}$ - and $\mathrm{y}$-axis indicates that the intensities at these wavelengths are bound together (they increase or decrease together). Negative correlation (blue color) means that the pair of wavelengths is anti-correlated: when the intensity of one wavelength increases, the other decreases (and vice versa). The computed correlation map based on experimental data of the generated SC reveals fine structure which is similar to reported earlier ${ }^{24}$. The clearly visible chessboard pattern multiplies the spectral correlation pattern of pump laser line related to wavelength jitter. The correlation map shows that the self-phase modulation (SPM), the stimulated Raman scattering (SRS) and the four-wave mixing (FMW) processes are responsible for 


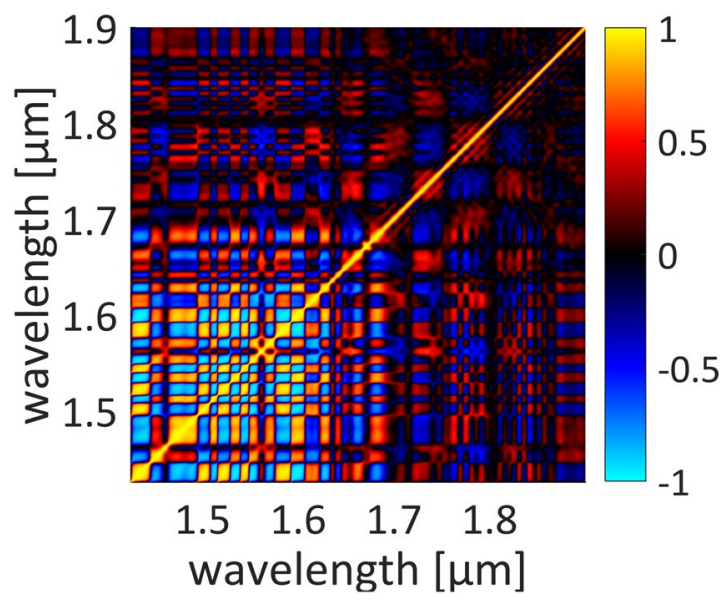

Figure 8. Spectral correlation maps obtained with DFT for the investigated all-fiber ANDi-SC.

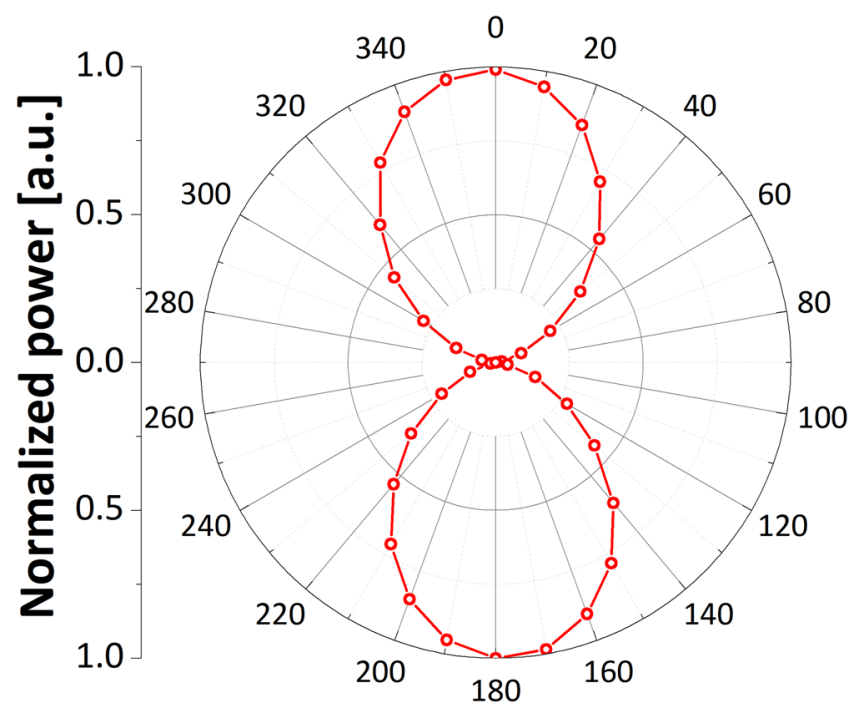

Figure 9. Polar plot indicating the high polarization extinction ratio of the generated SC.

spectral broadening. The SRS and FWM processes dominates SPM beyond $1.65 \mu \mathrm{m}$ as the correlation map is smoothed out for those wavelengths. The mostly checker-pattern-like appearance in the correlation map indicates wavelength jittering present in the supercontinuum, which might result from drifts in the pump laser. Since the entire system is free-running (without any active or passive stabilization of the oscillator or amplifier), we believe that this might be further optimized by applying e.g. thermal stabilization of the laser or improve the $980 \mathrm{~nm}$ pump stabilization.

Finally, we characterized experimentally the polarization state of the generated SC spectrum. Figure 9. presents the normalized output power as a function of orientation of a broadband polarizer placed after the SC output. The results confirm that spectrum is linearly polarized with polarization extinction ratio (PER) at the level of $1: 57(\sim 18 \mathrm{~dB})$

\section{Methods}

Er-doped fiber laser. All fibers in the laser cavity were spliced using a standard arc-fusion splicer (Fujikura FSM-100P). The EDF is Liekki Er80-4/125-PM and the passive fibers are Fujikura SM15-PS-U25D or equivalent. The output radiation of the laser was characterized with an optical spectrum analyzer (OSA, Yokogawa AQ6375) and an autocorrelator (APE PulseCheck).

Dispersive fourier transform measurement. The DFT was performed in an unequal-path Michelson interferometer similar to that presented in ${ }^{24,29}$, based on a $50 / 50$ splitter (designed for $1550 \mathrm{~nm}$ ). As a dispersive medium for DFT, a 500-m-long segment of SM2000 fiber (FIBRAIN Ltd.) was used, which is superior to SMF-28 in terms of long-wavelength transmission. The DFT results were recorded using an OSA (Yokogawa AQ6375) and $6 \mathrm{GHz}$ bandwidth oscilloscope (Agilent Infinuum DSO90604A) coupled with a $13 \mathrm{GHz}$ bandwidth InGaAs photodetector (Discovery Semiconductors DSC2-50S). 


\begin{tabular}{|l|l|l|l|}
\hline Subpulse & $\begin{array}{l}\text { normalized } \\
\text { amplitude } \mathrm{E}^{(\mathbf{i})}[\mathbf{1}]\end{array}$ & $\begin{array}{l}\text { pulse width } \\
\mathbf{T}^{(\mathbf{i})}[\mathbf{f s}]\end{array}$ & $\begin{array}{l}\text { pulse delay } \\
\mathbf{t}^{(\mathbf{i}}[\mathbf{f s}]\end{array}$ \\
\hline A & 1.0000 & 16.5 & - \\
\hline B & 0.2421 & 13 & 72 \\
\hline C & 0.1698 & 27 & 127 \\
\hline
\end{tabular}

Table 1. Fitted parameters of the pump pulse.

Nonlinear simulations of supercontinuum generation. The calculations were based on solving the NLSE via split-step Fourier method ${ }^{25-27}$ with self-developed software. In the nonlinear simulations we used the fiber properties given in Fig. 4 (group velocity dispersion, and effective mode area). We used calculated group velocity as data are needed for the NLSE solver in a broad range. We also used the fiber loss given in ${ }^{12}$. In order to estimate the pulse shape for nonlinear simulations, we performed fitting of intensity autocorrelation with assumed pulse shape given with $\mathrm{Eq}(1)$ :

$$
E(t)=E^{(A)} \operatorname{sech}\left(\frac{t}{T^{(A)}}\right)+E^{(B)} \operatorname{sech}\left(\frac{t-t^{(B)}}{T^{(B)}}\right)+E^{(C)} \operatorname{sech}\left(\frac{t-t^{(C)}}{T^{(C)}}\right)
$$

The pulse is described as the sum of three subpulses: main (A) and two additional (B and C). The additional subpulses are introduced to account a small pedestal observed in the autocorrelation trace. The fitting parameters are summarized in Table 1.

In the simulations, we set the input pulse described with Eq. (1) with parameters given in Table 1. We estimated the average power of input pulse to $72 \mathrm{~mW}$ - for this input power, the calculated output power was $57 \mathrm{~mW}$ - in agreement with the measurements. This way, we estimated the coupling efficiency to $62 \%$ (in agreement with measured splice loss).

\section{Summary}

To summarize, we have reported the first, to our knowledge, entirely fiberized source of coherent and linearly polarized octave-spanning supercontinuum generated in an all-normal dispersion fiber. The silica ANDi fiber is pumped at $1.55 \mu \mathrm{m}$ by an ultrasimple Er-doped fiber laser, resulting in a broad SC spanning from $1.1 \mu \mathrm{m}$ to $2.2 \mu \mathrm{m}$. The average output power of the SC is at the level of $57 \mathrm{~mW}$. We have confirmed the high coherence of the SC by means of dispersive Fourier transform and spectral interference between consecutive pulses of the SC train. We believe that the excellent coherence properties connected with unprecedented simplicity, compactness, low-cost, and lack of any moving parts makes the presented SC source attractive for practical applications in outside-laboratory conditions.

\section{References}

1. Ranka, J. K., Windeler, R. S. \& Stentz, A. J. Visible continuum generation in air-silica microstructure optical fibers with anomalous dispersion at $800 \mathrm{~nm}$. Opt. Lett. 25, 25-27 (2000).

2. Hori, T., Takayanagi, J., Nishizawa, N. \& Goto, T. Flatly broadened, wideband and low noise supercontinuum generation in highly nonlinear hybrid fiber. Opt. Express 12, 317-324 (2004).

3. Nishizawa, N. \& Takayanagi, J. Octave spanning high-quality supercontinuum generation in all-fiber system. J. Opt. Soc. Am. B 24, 1786-1792 (2007).

4. Heidt, A. M. Pulse preserving flat-top supercontinuum generation in all-normal dispersion photonic crystal fibers. J. Opt. Soc. Am. B 27, 550-559 (2010).

5. Finot, C., Kibler, B., Provost, L. \& Wabnitz, S. Beneficial impact of wave-breaking for coherent continuum formation in normally dispersive nonlinear fibers. J. Opt. Soc. Am. B 25, 1938-1948 (2008).

6. Heidt, A. M. et al. Coherent octave spanning near-infrared and visible supercontinuum generation in all-normal dispersion photonic crystal fibers. Opt. Express 19, 3775-3787 (2011).

7. Martynkien, T., Pysz, D., Stępień, R. \& Buczyński, R. All-solid microstructured fiber with flat normal chromatic dispersion. Opt. Lett. 39, 2342-2345 (2014).

8. Klimczak, M. et al. Coherent supercontinuum generation up to $2.3 \mu \mathrm{m}$ in all-solid soft-glass photonic crystal fibers with flat allnormal dispersion. Opt. Express 22, 18824-18832 (2014).

9. Kedenburg, S., Steinle, T., Mörz, F., Steinmann, A. \& Giessen, H. High-power mid-infrared high repetition-rate supercontinuum source based on a chalcogenide step-index fiber. Opt. Lett. 40, 2668-2671 (2015).

10. Zhao, Z. et al. 1.5-14 $\mu \mathrm{m}$ midinfrared supercontinuum generation in a low-loss Te-based chalcogenide step-index fiber. Opt. Lett. 41, 5222-5225 (2016).

11. Tarnowski, K. \& Urbanczyk, W. All-normal dispersion hole-assisted silica fibers for generation of supercontinuum reaching midinfrared. IEEE Photonics J. 8, 7100311 (2016).

12. Tarnowski, K. et al. Coherent supercontinuum generation up to $2.2 \mu \mathrm{m}$ in an all-normal dispersion microstructured silica fiber. Opt. Express 24, 30523-30536 (2016).

13. Tarnowski, K. et al. Polarized all-normal dispersion supercontinuum reaching $2.5 \mu \mathrm{m}$ generated in a birefringent microstructured silica fiber. Opt. Express 25, 27452-27463 (2017).

14. Shreesha Rao, D. S. et al. Ultra-low-noise supercontinuum generation with a flat near-zero normal dispersion fiber. Opt. Lett. 44, 2216-2219 (2019).

15. Gao, W. et al. All-fiber broadband supercontinuum source with high efficiency in a step-index high nonlinear silica fiber. Appl. Opt. 51, 1071-1075 (2012).

16. Hong-Wei, C. et al. Generation of a compact high-power high-efficiency normal-dispersion pumping supercontinuum in silica photonic crystal fiber pumped with a 1064-nm picosecond pulse. Chin. Phys. B 22, 064211 (2013).

17. Okamura, A., Sakakibara, Y., Omoda, E., Kataura, H. \& Nishizawa, N. Experimental analysis of coherent supercontinuum generation and ultrashort pulse generation using cross-correlation frequency resolved optical gating (X-FROG). J. Opt. Soc. Am. B 32, 400-406 (2015). 
18. Nozaki, Y., Nomura, Y., Aramaki, M. \& Nishizawa, N. Octave spanning coherent supercontinuum generation using 51 fs high-power ultrashort pulse from Er-doped similariton amplifier. Jpn. J. Appl. Phys. 53, 8-11 (2014).

19. Lindfors, K., Kalkbrenner, T., Stoller, P. \& Sandoghdar, V. Detection and spectroscopy of gold nanoparticles using supercontinuum white light confocal microscopy. Phys. Rev. Lett. 93, 037401 (2004).

20. Hartl, I. et al. Ultrahigh-resolution optical coherence tomography using continuum generation in an air-silica microstructure optical fiber. Opt. Lett. 26, 608-610 (2001).

21. Inaba, H. et al. Long-term measurement of optical frequencies using a simple, robust and low-noise fiber based frequency comb. Opt. Express 14, 5223-5231 (2006).

22. Sotor, J. \& Sobon, G. 24 fs and $3 \mathrm{~nJ}$ pulse generation from a simple, all polarization maintaining Er-doped fiber laser. Laser Phys. Lett. 13, $125102(2016)$.

23. Goda, K. \& Jalali, B. Dispersive Fourier transformation for fast continuous single-shot measurements. Nat. Photonics 7, 102-112 (2013).

24. Klimczak, M., Soboń, G., Kasztelanic, R., Abramski, K. M. \& Buczyński, R. Direct comparison of shot-to-shot noise performance of all normal dispersion and anomalous dispersion supercontinuum pumped with sub-picosecond pulse fiber-based laser. Sci. Rep. 6, 1-14 (2016).

25. Dudley, J. M., Genty, G. \& Coen, S. Supercontinuum generation in photonic crystal fiber. Rev. Mod. Phys. 78, 1135-1184 (2006)

26. Dudley J. M. \& Taylor, J. R. Supercontinuum Generation in Optical Fibers (Cambridge University Press, 2010).

27. Agrawal, G. P. Nonlinear Fiber Optics, 5th Edition (Academic Press, 2012).

28. Nicholson, J. W. \& Yan, M. F. Cross-coherence measurements of supercontinua generated in highly-nonlinear, dispersion shifted fiber at $1550 \mathrm{~nm}$. Opt. Express 12, 679-688 (2004).

29. Runge, A. F. J., Aguergaray, C., Broderick, N. G. R. \& Erkintalo, M. Coherence and shot-to-shot spectral fluctuations in noise-like ultrafast fiber lasers. Opt. Lett. 38, 4327-4330 (2013).

30. Godin, T. et al. Real time noise and wavelength correlations in octave-spanning supercontinuum generation. Opt. Express 21, 18452-18460 (2013).

\section{Acknowledgements}

The work presented in this publication was funded by the Foundation for Polish Science FNP (First TEAM/20174/39) and partially by the Faculty of Electronics, Wrocław University of Science and Technology (0401/0030/18). We would like to express our gratitude to FIBRAIN Ltd. for providing the SM2000 fiber used in the DFT experiments. We are very thankful to Robert Lindberg (KTH, Sweden) for his invaluable help in the interpretation of the correlation maps.

\section{Author Contributions}

K.T. and G.S. conceived the study. K.T. designed the nonlinear fiber, performed the nonlinear simulations, and processed all the experimental data. T.M. measured the dispersion of the nonlinear fiber and contributed to the data analysis. P.M. fabricated the nonlinear fiber. J.S. designed and built the Er-doped fiber laser. G.S. set-up the DFT and coherence experiments and performed all experimental measurements of SC generation. All Authors contributed to writing of the manuscript.

\section{Additional Information}

Competing Interests: The authors declare no competing interests.

Publisher's note: Springer Nature remains neutral with regard to jurisdictional claims in published maps and institutional affiliations.

(c) (i) Open Access This article is licensed under a Creative Commons Attribution 4.0 International License, which permits use, sharing, adaptation, distribution and reproduction in any medium or format, as long as you give appropriate credit to the original author(s) and the source, provide a link to the Creative Commons license, and indicate if changes were made. The images or other third party material in this article are included in the article's Creative Commons license, unless indicated otherwise in a credit line to the material. If material is not included in the article's Creative Commons license and your intended use is not permitted by statutory regulation or exceeds the permitted use, you will need to obtain permission directly from the copyright holder. To view a copy of this license, visit http://creativecommons.org/licenses/by/4.0/.

(c) The Author(s) 2019 